\title{
Design and Implementation of Booth Multiplier using Approximate Adders
}

\author{
Shital Keskar ${ }^{1}$, Prof T. S. Mote ${ }^{2}$ \\ ENTC Department, Pune University, Pune, India ${ }^{1,2}$,
}

\begin{abstract}
Low power is an imperative requirement for portable multimedia devices employing various signal processing algorithms and architectures. In most multimedia applications, human beings can gather useful information from slightly erroneous outputs. Therefore, we do not need to produce exactly correct numerical outputs. Previous research in this context exploits error resiliency primarily through voltage overscaling, utilizing algorithmic and architectural techniques to mitigate the resulting errors. In this paper, we propose logic complexity reduction at the transistor level as an alternative approach to take advantage of the relaxation of numerical accuracy. We demonstrate this concept by proposing various imprecise or approximate full adder cells with reduced complexity at the transistor level, and utilize them to design approximate multi-bit adders. In addition to the inherent reduction in switched capacitance, our techniques result in significantly shorter critical paths, enabling voltage scaling.
\end{abstract}

Keyword: Approximate computing, low power, mirror adder. Booths multiplier, Wallace tree multiplier

\section{INTRODUCTION}

Digital signal processing (DSP) blocks form the backbone of various multimedia applications used in portable devices. Most of these DSP blocks implement image and video processing algorithms, where the ultimate output is either an image or a video for human consumption. Human beings have limited perceptual abilities when interpreting an image or a video. This allows the outputs of these algorithms to be numerically approximate rather than accurate. This relaxation on numerical exactness provides some freedom to carry out imprecise or approximate computation. We can use this freedom to come up with low-power designs at different levels of design abstraction, namely, logic, architecture, and algorithm.

The paradigm of approximate computing is specific to select hardware implementations of DSP blocks. It is shown in [1] that an embedded reduced instruction set computing processor consumes $70 \%$ of the energy in supplying data and instructions, and 6\% of the energy while performing arithmetic only. Therefore, using approximate arithmetic in such a scenario will not provide much energy benefit when considering the complete processor. Programmable processors are designed for general-purpose applications with no application-specific specialization.

Therefore, there may not be many applications that will be able to tolerate errors due to approximate computing. This also makes general-purpose processors not suited for using approximate building blocks. This issue has already been discussed in [13]. Therefore, in this paper, we consider application-specific integrated circuit implementations of error-resilient applications like image and video compression.

We target the most computationally intensive blocks in these applications and build them using approximate hardware to show substantial improvements in power consumption with little loss in output quality.
Few works that focus on low-power design through approximate computing at the algorithm and architecture levels include algorithmic noise tolerance (ANT) [3]-[6], significance driven computation (SDC) [7]-[9], and nonuniform voltage overscaling (VOS) [10]. All these techniques are based on the central concept of VOS, coupled with additional circuitry for correcting or limiting the resulting errors. In [11], a fast but "inaccurate" adder is proposed. It is based on the idea that on average, the length of the longest sequence of propagate signals is approximately $\log n$, where $n$ is the bitwidth of the two integers to be added. An error-tolerant adder is proposed in [12] that operates by splitting the input operands into accurate and inaccurate parts. However, neither of these techniques target logic complexity reduction. Powerefficient multiplier architecture is proposed in [13] that uses a $2 \times 2$ inaccurate multiplier block resulting from Karnaugh map simplification. This paper considers logic complexity reduction using Karnaugh maps. Shin and Gupta [14] and Phillips et al. [15] also proposed logic complexity reduction by Karnaugh map simplification. Other works that focus on logic complexity reduction at the gate level are [16]-[19]. Other approaches use complexity reduction at the algorithm level to meet realtime energy constraints [20], [21].

Previous works on logic complexity reduction have focused on algorithm, logic, and gate levels. We propose logic complexity reduction at the transistor level. We apply this to addition at the bit level by simplifying the mirror adder (MA) circuit. We develop imprecise but simplified arithmetic units, which provide an extra layer of power savings over conventional low-power design techniques. This is attributed to the reduced logic complexity of the proposed approximate arithmetic units. Note that the approximate arithmetic units not only have a reduced number of transistors, but care is taken to ensure that the internal node capacitances are greatly reduced. Complexity reduction leads to power reduction in two 
different ways. First, an inherent reduction in switched capacitance and leakage results from having smaller hardware. Second, complexity reduction frequently leads to shorter critical paths, facilitating voltage reduction without any timing-induced errors. In summary, our work significantly differs from other works (SDC, ANT, and nonuniform VOS) since we adopt a different approach for exploiting error resiliency. Our aim is to target low-power design using simplified and approximate logic implementations. Since DSP blocks mainly consist of adders and multipliers (which are, in turn, built using adders), we propose several approximate adders, which can be used effectively in such blocks.

A preliminary version of our work appeared in [22]. We extend our paper in [22] by providing two more simplified versions of the MA. Furthermore, we propose a measure of the "quality" of a DSP block that uses approximate adders. We also propose a methodology that can be used to harness maximum power savings using approximate adders, subject to a specific quality constraint. Our contributions in this paper can be summarized as follows.

1) We propose logic complexity reduction at the transistor level as an alternative approach to approximate computing for DSP applications.

2) We show how to simplify the logic complexity of a conventional MA cell by reducing the number of transistors and internal node capacitances. Keeping this aim in mind, we propose five different simplified versions of the MA, ensuring minimal errors in the full adder (FA) truth table.

3) We utilize the simplified versions of the FA cell to propose several imprecise or approximate multi-bit adders that can be used as building blocks of DSP systems. To maintain a reasonable output quality, approximate FA cells used only in the least significant bits (LSBs). We particularly focus on adder structures that use FA cells as their basic building blocks.

4) VOS is a very popular technique to get large improvements in power consumption. However, VOS will lead to delay failures in the most significant bits (MSBs). This might lead to large errors in corresponding outputs and severely mess up the output quality of the application. We use approximate FA cells only in the LSBs, while the MSBs use accurate FA cells. Therefore, at iso frequency, the errors introduced by VOS will be much higher, when compared to proposed approximate adders. Since truncation is a well-known technique to facilitate voltage scaling,. In general, our approach may be applied to any arithmetic circuit built with FAs.

\section{APPROXIMATE ADDERS}

In this section, we discuss different methodologies for designing approximate adders. Since the MA [23] is one of the widely used economical implementations of an FA
[24], we use it as our basis for proposing different approximations of an FA cell.

Approximation Strategies for the MA

In this section, we explain step-by-step procedures for coming up with various approximate MA cells with fewer transistors. Removal of some series connected transistors will facilitate faster charging/discharging of node capacitances. Moreover, complexity reduction by removal of transistors also aids in reducing the $\alpha C$ term (switched capacitance) in the dynamic power expression $P$ dynamic $=$ $\alpha C V 2 \mathrm{DD} f$, where $\alpha$ is the switching activity or average number of switching transitions per unit time and $C$ is the load capacitance being charged/discharged. This directly results in lower power dissipation. Area reduction is also achieved by this process. Now, let us discuss the conventional MA implementation followed by the proposed approximations used economical implementations of an FA [24], we use it as our basis for proposing different approximations of an FA cell.

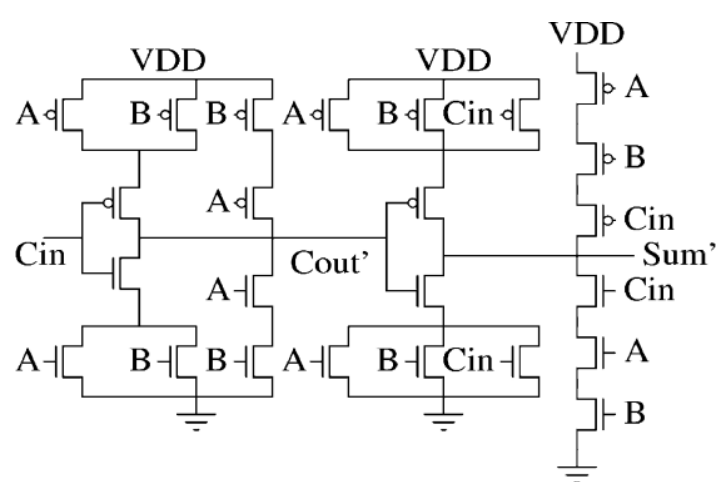

Fig. 1. Conventional MA.

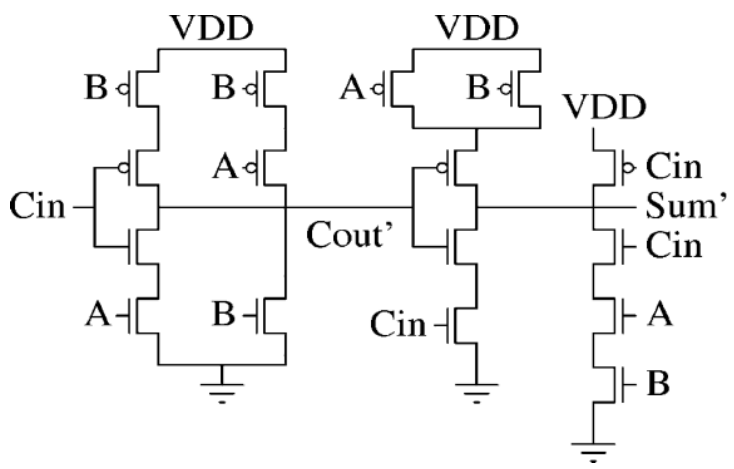

Fig. 2. MA approximation 1.

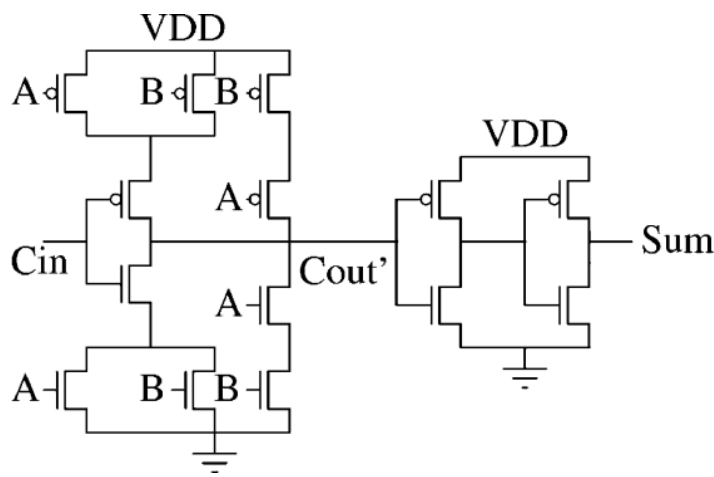

Fig. 3. MA approximation 2. 


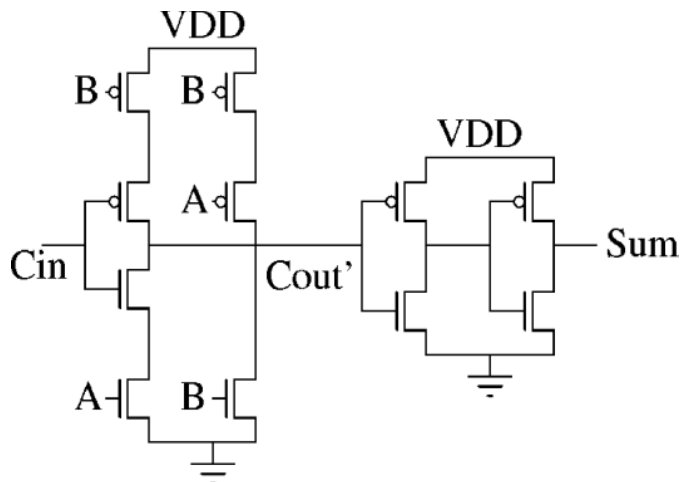

Fig. 4. MA approximation 3.

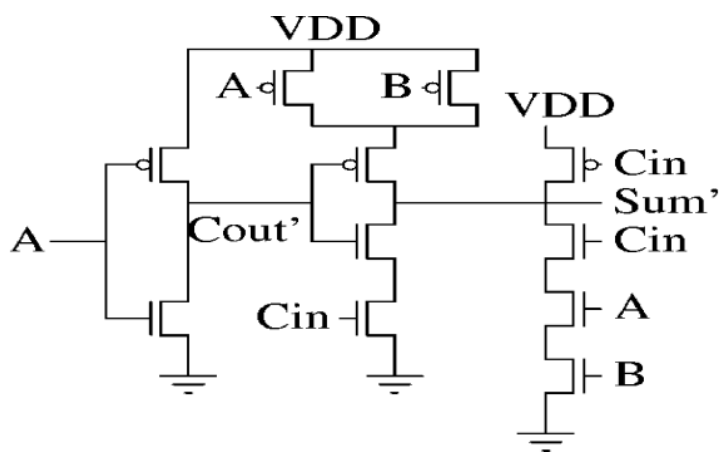

Fig. 5. MA approximation 4.

1) Conventional MA: Fig. 1 shows the transistor-level schematic of a conventional MA [23], which is a popular way of implementing an FA. It consists of a total of 24 transistors. Since this implementation is not based on complementary CMOS logic, it provides a good opportunity to design an approximate version with removal of selected transistors.

2) Approximation 1: In order to get an approximate MA with fewer transistors, we start to remove transistors from the conventional schematic one by one. However, we cannot do this in an arbitrary fashion. We need to make sure that any input combination of
A,B and Cin does not result in short circuits or open circuits in the simplified schematic. Another important criterion is that the resulting simplification should introduce minimal errors in the FA truth table. A judicious selection of transistors to be removed (ensuring no open or short circuits) results in a schematic shown in Fig. 2, which we call approximation 1. Clearly, this schematic has eight fewer transistors compared to the conventional MA schematic. In this case, there is one error in Cout and two errors in Sum, as shown in Table I. A tick mark denotes a match with the corresponding accurate output and a cross denotes an error.

3) Approximation 2: The truth table of an FA shows that Sum $=$ Cout 1 for six out of eight cases, except for the input combinations $\mathrm{A}=0, \mathrm{~B}=0, \mathrm{Cin}=0$ and $\mathrm{A}=1, \mathrm{~B}$ $=1, \mathrm{Cin}=1$. Now, in the conventional MA, Cout is computed in the first stage. Thus, an easy way to get a simplified schematic is to set Sum $=$ Cout. However, we introduce a buffer stage after Cout (see Fig. 3) to implement the same functionality.

The reason for this can be explained as follows. If we set $\mathrm{Sum}=$ Cout as it is in the conventional MA, the total capacitance at the Sum node would be a combination of four source-drain diffusion and two gate capacitances. This is a considerable increase compared to the conventional case or approximation 1. Such a design would lead to a delay penalty in cases where two or more multi-bit approximate adders are connected in series, which is very common in DSP applications. Fig. 3 shows the schematic obtained using the above approach. We call this approximation

2. Here, Sum has only two errors, while Cout is correct for all cases, as shown in Table I.

Table 1: Truth Table for Conventional Adder and Approximations 1 to 4

\begin{tabular}{|c|c|c|c|c|c|c|c|c|c|c|c|c|}
\hline \multicolumn{3}{|c|}{ Inputs } & \multicolumn{2}{|c|}{ Accurate Outputs } & \multicolumn{8}{|c|}{ Approximate Outputs } \\
\hline A & $B$ & $C_{\text {in }}$ & Sum & $C_{\text {out }}$ & Sum $_{1}$ & $C_{\text {outl }}$ & $\mathrm{Sum}_{2}$ & $C_{\text {out2 }}$ & $\mathrm{Sum}_{3}$ & $C_{\text {out3 }}$ & Sum. & $C_{\text {out } 4}$ \\
\hline 0 & 0 & 0 & 0 & 0 & $0 \sqrt{ }$ & $0 \sqrt{ }$ & $1 \times$ & $0 \sqrt{ }$ & $1 x$ & $0 \sqrt{ }$ & $0 \sqrt{ }$ & $0 \sqrt{ }$ \\
\hline 0 & 0 & 1 & 1 & 0 & $1 \sqrt{ }$ & $0 \sqrt{ }$ & $1 \sqrt{ }$ & $0 \sqrt{ }$ & $1 \sqrt{ }$ & $0 \sqrt{ }$ & $1 \sqrt{ }$ & $0 \sqrt{ }$ \\
\hline 0 & 1 & 0 & 1 & 0 & $0 \times$ & $1 \times$ & $1 \sqrt{ }$ & $0 \sqrt{ }$ & $0 x$ & $1 x$ & $0 \times$ & $0 \sqrt{ }$ \\
\hline 0 & 1 & 1 & 0 & 1 & $0 \sqrt{ }$ & $1 \sqrt{ }$ & $0 \sqrt{ }$ & $1 \sqrt{ }$ & $0 \sqrt{ }$ & $1 \sqrt{ }$ & $1 x$ & $0 \times$ \\
\hline 1 & 0 & 0 & 1 & 0 & $0 \times$ & $0 \sqrt{ }$ & $1 \sqrt{ }$ & $0 \sqrt{ }$ & $1 \sqrt{ }$ & $0 \sqrt{ }$ & $0 \times$ & $1 \times$ \\
\hline 1 & 0 & 1 & 0 & 1 & $0 \sqrt{ }$ & $1 \sqrt{ }$ & $0 \sqrt{ }$ & $1 \sqrt{ }$ & $0 \sqrt{ }$ & $1 \sqrt{ }$ & $0 \sqrt{ }$ & $1 \sqrt{ }$ \\
\hline 1 & 1 & 0 & 0 & 1 & $0 \sqrt{ }$ & $1 \sqrt{ }$ & $0 \sqrt{ }$ & $1 \sqrt{ }$ & $0 \sqrt{ }$ & $1 \sqrt{ }$ & $0 \sqrt{ }$ & $1 \sqrt{ }$ \\
\hline 1 & 1 & 1 & 1 & 1 & $1 \sqrt{ }$ & $1 \sqrt{ }$ & $0 \times$ & $1 \sqrt{ }$ & $0 \times$ & $1 \sqrt{ }$ & $1 \sqrt{ }$ & $1 \sqrt{ }$ \\
\hline
\end{tabular}




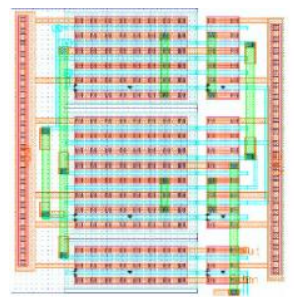

Conventional
Fig 6. Layouts of Conventional MA and Approximation Cell

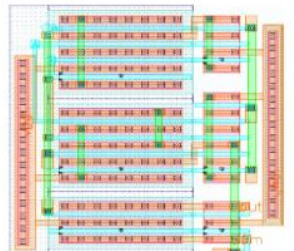

Approximation 1

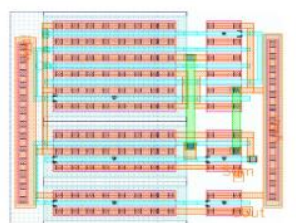

Approximation 2

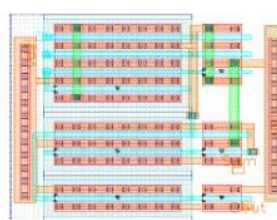

Approximation 3

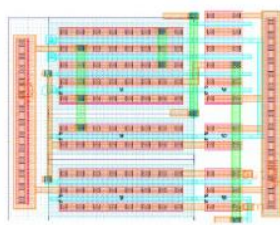

Approximation 4

Table 3 Experimental Results for one bit adder

\begin{tabular}{|c|c|c|c|}
\hline & $\begin{array}{c}\text { Power Consumption( } \\
\mu \mathrm{w})\end{array}$ & $\begin{array}{c}\text { Layout Area( } \\
\left.\mu \mathrm{m}^{2}\right)\end{array}$ & Delay(ps) \\
\hline Conventional MA & 148 & $54^{*} 12$ & 15 \\
\hline Approximation 1 & 72.682 & $36^{*} 11$ & 10 \\
\hline Approximation 2 & 9.098 & $32 * 12$ & 20 \\
\hline Approximation 3 & 8.782 & $26^{*} 12$ & 21 \\
\hline Approximation 4 & 7.394 & $25^{*} 11$ & 15 \\
\hline
\end{tabular}

Table 4 Experimental Results for eight bit booths multiplier

\begin{tabular}{|c|c|c|c|c|c|}
\hline & $\begin{array}{c}\text { Power( } \\
\mathrm{mw})\end{array}$ & Delay(ps) & $\begin{array}{c}\text { No of Adders } \\
\text { used in CLA }\end{array}$ & $\begin{array}{c}\text { Total No of } \\
\text { Transistors used in } \\
\text { CLA }\end{array}$ & $\begin{array}{c}\text { Layout Area for } \\
\text { CLA } \\
\left(\mu \mathrm{m}^{2}\right. \\
)\end{array}$ \\
\hline Conventional MA & 585.4 & 551.1 & 15 & 360 & 180 \\
\hline Approximation1 & 584.9 & 551.08 & 15 & 248 & 124 \\
\hline Approximation2 & 601.7 & 551.09 & 15 & 218 & 109 \\
\hline Approximation3 & 592.9 & 551.27 & 15 & 176 & 88 \\
\hline Approximation4 & 582.6 & 551.09 & 15 & 178 & 89 \\
\hline
\end{tabular}

Table 2 Choosing Approximation 5

\begin{tabular}{|l|c|c|c|}
\hline \multicolumn{2}{|c|}{ Choice 1} & \multicolumn{2}{c|}{ Choice 2} \\
\hline Sum= $A$ & $C_{\text {out }}=A$ & Sum= $B$ & $C_{\text {out }}=A$ \\
\hline $0 \checkmark$ & $0 \checkmark$ & $0 \checkmark$ & $0 \checkmark$ \\
\hline $0 \times$ & $0 \checkmark$ & $0 \times$ & $0 \checkmark$ \\
\hline $0 \times$ & $0 \checkmark$ & $1 \checkmark$ & $0 \checkmark$ \\
\hline $0 \checkmark$ & $0 \times$ & $1 \times$ & $0 \times$ \\
\hline $1 \checkmark$ & $1 \times$ & $0 \times$ & $1 \times$ \\
\hline $1 \times$ & $1 \checkmark$ & $0 \checkmark$ & $1 \checkmark$ \\
\hline $1 \times$ & $1 \checkmark$ & $1 \times$ & $1 \checkmark$ \\
\hline $1 \checkmark$ & $1 \checkmark$ & $1 \checkmark$ & $1 \checkmark$ \\
\hline
\end{tabular}

4) Approximation 3: Further simplification can be obtained by combining approximations 1 and 2. Note that this introduces one error in Cout and three errors in Sum, as shown in Table I. The corresponding simplified schematic is shown in Fig. 4.

5) Approximation 4: A close observation of the FA truth table shows that Cout $=$ A for six out of eight cases. Similarly, Cout = B for six out of eight cases. Since A and $\mathrm{B}$ are interchangeable, fourth approximation where we just use an inverter with input A to calculate Cout and Sum is calculated similar to approximation 1. This introduces two errors in Cout and three errors in as shown in Table I. The corresponding simplified schematic is shown in Fig. 5. In all we consider Cout
= A. Thus, we propose a Sum, using an inverter with Cout as input.

6) Approximation 5: In approximation 4, we find that there are three errors in Sum. We extend this approximation by allowing one more error, i.e., four errors in Sum. We use the approximation Cout = A, as in approximation 4. If we want to make Sum independent of Cin, we have two choices, Sum= A and Sum= B. Thus, we have two alternatives for approximation 5, namely, $\mathrm{Sum}=\mathrm{A}$, Cout $=\mathrm{A}$ and Sum $=$ B, Cout $=$ A, which are shown in Table 2. The table shows which entries match with and differ from the corresponding accurate outputs (shown by tick marks and crosses). If we observe choice 1, we find that both Sum and Cout match with accurate outputs in only two out of eight cases. In choice 2, Sum and Cout match with accurate outputs in four out of eight cases. Therefore, to minimize errors both in Sum and Cout, we go for choice 2 as approximation 5. Our main thrust here is to ensure that for a particular input combination (A,B and Cin), ensuring correctness in Sum also makes Cout correct. Now consider the addition of two 20-b integers a[19:0] and b[19:0] using an RCA. Suppose we use approximate FAs for 7 LSBs. Then, Cin[7] = Cout[6]. Note that Cout[6] is approximate. Applying this approximation to our present example, we find that carry propagation from bit 0 to bit 6 is entirely eliminated. In addition, the 
circuitry needed to calculate Cout[0] to Cout[5] is also saved. To limit the output capacitance at Sum and Cout nodes, we implement approximation 5: Sum= B, Cout $=$ A using buffers.

Layouts of conventional MA and different approximations in IBM 90-nm technology are shown in Fig. 6. Layout area the conventional MA and different approximations are compared in Table III. Approximation 5 uses only buffers. The layout area of a single buffer is $6.77 \mu \mathrm{m} 2$.

7) Boots multiplier: Multiplication is a most commonly used operation in many computing systems. Intact multiplication is nothing but addition since, multiplicand adds to itself multiplier number of times gives the multiplication value between multiplier and multiplicand. But considering the fact that this kind of implementation really takes huge hardware resources and the circuit operates at utterly low speed. If we observe closely multiplication operation involves two steps one is producing partial products and adding these partial products. Thus, the speed of a multiplier hardly depends on how fast generate the partial products and how fast we can add them together. If the number of partial products to be generated is of less than it is indirectly means that we have achieved the speed in generating partial products. To speed up the addition among the partial products we need fast adder architectures. The high speed Booth multiplier is used for digital signal processing (DSP) applications such as for multimedia and communication systems. We designed and implemented a multiplier unit that can perform multiplication operation on both positive and negative for signed numbers.

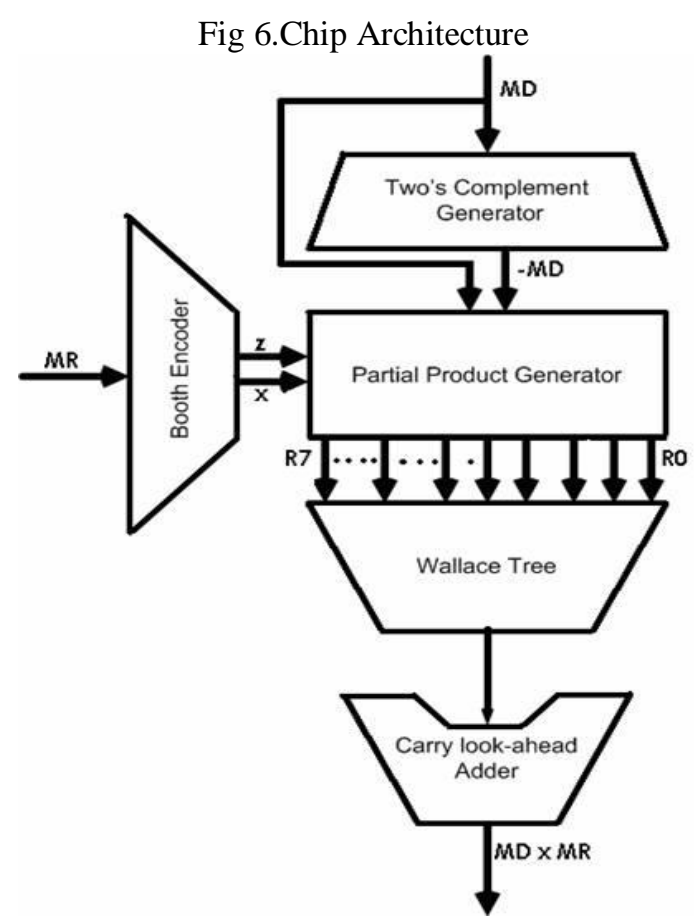

Algorithm: The architecture consists of five parts: two's complement Generator, Booth Encoder, Partial Product, Wallace tree and Carry Look-ahead Adder. In implementation of booths algorithm there are two operand multiplier and multiplicand.

8)Wallace Tree Multiplier: To improving the speed of the parallel multiplier Wallace multiplier is used. Wallace introduced a very important iterative realization of parallel multiplier. Parallel multipliers can reduce the number of partial product rows to be added by half, thus reducing the size and enhance the speed of the tree. This advantage becomes more pronounced for multipliers of bigger than 16 bits. In Wallace tree architecture, all the bits of all of the partial products in each column are added together by a set of counters in parallel without propagating any carries. Another set of counters then reduces this new matrix and so on, until a two-row matrix is generated. The most common counter used is the 3:2 counters which is a Full Adder. The final results are added using usually carry look ahead adder. A block diagram of 4 bit Wallace Tree multiplier is shown in below. As seen from the block diagram partial products are added in Wallace tree block. The result of these additions is the final product bits and sum and carry bits which are added in the final carry look ahead adder.

Power consumption and booths multiplication output of: 1.

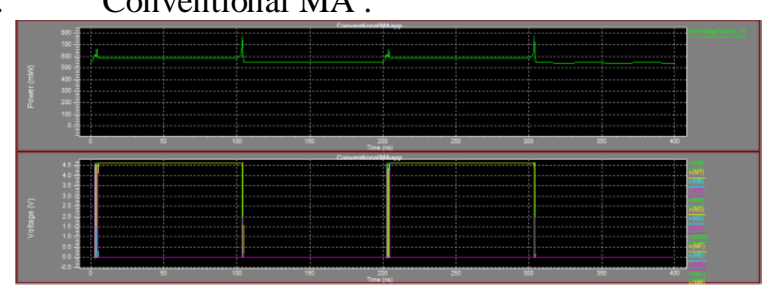

2. Approximation1:

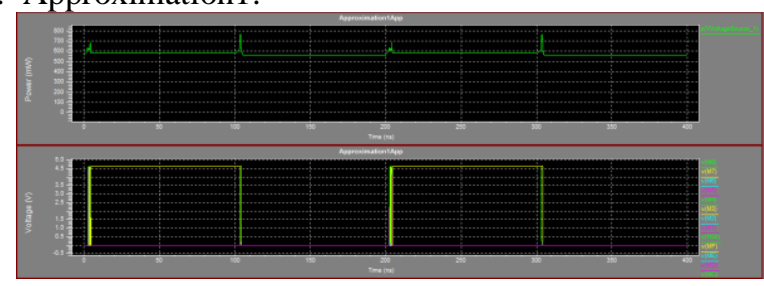

3.Approximation 2:

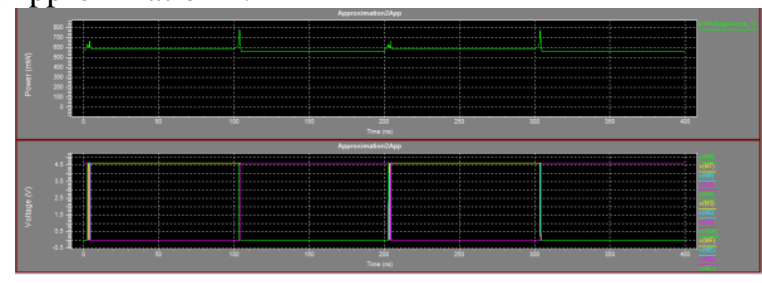

4. Approximation 3:

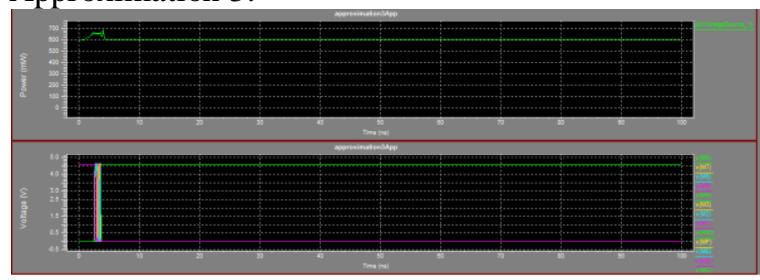




\section{Approximation 4:}

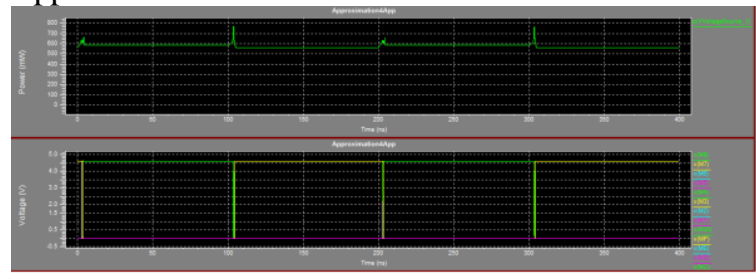

\section{CONCLUSION}

In this paper, we proposed several imprecise or approximate adders that can be effectively utilized to trade off power and quality for error-resilient DSP systems. Our approach aimed to simplify the complexity of a conventional MA cell by reducing the number of transistors and also the load capacitances. When the errors introduced by these approximations were reflected at a high level in a typical DSP algorithm, the impact on output quality was very little. Note that our approach differed from previous approaches where errors were introduced due to VOS. A decrease in the number of series connected transistors helped in reducing the effective switched capacitance and achieving voltage scaling. We present a 8bit $\times 8$-bit multiplier capable of carrying signed operations on positive and negative operand. The proposed approximations are used for multiplier design and optimized in terms of delay, power consumption and area.

\section{REFERENCES}

[1] W. Dally, J. Balfour, D. Black-Shaffer, J. Chen, R. Harting, V. Parikh,J. Park, and D. Sheffield, "Efficient embedded computing," Computer,vol. 41, no. 7, pp. 27-32, Jul. 2008

[2] P. Kulkarni, P. Gupta, and M. Ercegovac, "Trading accuracy for power with an underdesigned multiplier architecture," in Proc. 24th IEEE Int.Conf. VLSI Design, Jan. 2011, pp. 346-351.

[3] R. Hegde and N. Shanbhag, "Energy-efficient signal processing via algorithmic noise-tolerance," in Proc. IEEE/ACM Int. Symp. Low Power Electron. Design, Aug. 1999, pp. 30-35.

[4] R. Hegde and N. R. Shanbhag, "Soft digital signal processing," IEEE Trans. Very Large Scale Integr. Syst., vol. 9, no. 6, pp. 813823, Jun.2001.

[5] B. Shim, S. Sridhara, and N. Shanbhag, "Reliable low-power digital signal processing via reduced precision redundancy," IEEE Trans. Very Large Scale Integr. Syst., vol. 12, no. 5, pp. 497-510, May 2004

[6] G. Varatkar and N. Shanbhag, "Energy-efficient motion estimation using error-tolerance," in Proc. IEEE/ACM Int. Symp. Low Power Electron.Design, Oct. 2006, pp. 113-118.

[7] D. Mohapatra, G. Karakonstantis, and K. Roy, "Significance driven computation: A voltage-scalable, variation-aware, quality-tuning motion estimator," in Proc. IEEE/ACM Int. Symp. Low Power Electron. Design, Aug. 2009, pp. 195-200.

[8] N. Banerjee, G. Karakonstantis, and K. Roy, "Process variation tolerant low power DCT architecture," in Proc. Design, Automat. Test Eur., 2007, pp. 1-6.

[9] G. Karakonstantis, D. Mohapatra, and K. Roy, "System level DSP synthesis using voltage overscaling, unequal error protection and adaptive quality tuning," in Proc. IEEE Workshop Signal Processing Systems, Oct. 2009, pp. 133-138.

[10] L. N. Chakrapani, K. K. Muntimadugu, L. Avinash, J. George, and K. V. Palem, "Highly energy and performance efficient embedded computing through approximately correct arithmetic: A mathematical foundation and preliminary experimental validation," in Proc. CASES, 2008, pp. 187-196.

[11] A. K. Verma, P. Brisk, and P. Ienne, "Variable latency speculative addition: A new paradigm for arithmetic circuit design," in Proc. Design, Automat. Test Eur., 2008, pp. 1250-1255.
[12] N. Zhu, W. L. Goh, and K. S. Yeo, "An enhanced low-power highspeed adder for error-tolerant application," in Proc. IEEE Int. Symp. Integr. Circuits, Dec. 2009, pp. 69-72.

[13] P. Kulkarni, P. Gupta, and M. D. Ercegovac, "Trading accuracy for power in a multiplier architecture," J. Low Power Electron., vol. 7, no. 4, pp. 490-501, 2011.

[14] D. Shin and S. K. Gupta, "Approximate logic synthesis for error tolerant applications," in Proc. Design, Automat. Test Eur., 2010, pp. 957-960.

[15] B. J. Phillips, D. R. Kelly, and B. W. Ng, "Estimating adders for a low density parity check decoder," Proc. SPIE, vol. 6313, p. 631302, Aug. 2006

[16] H. R. Mahdiani, A. Ahmadi, S. M. Fakhraie, and C. Lucas, "Bioinspired imprecise computational blocks for efficient VLSI implementation of soft-computing applications," IEEE Trans. Circuits Syst. Part I, vol. 57, no. 4, pp. 850-862, Apr. 2010.

[17] D. Shin and S. K. Gupta, "A re-design technique for datapath modules in error tolerant applications," in Proc. 17th Asian Test Symp., 2008, pp. 431-437.

[18] D. Kelly and B. Phillips, "Arithmetic data value speculation," in Proc. Asia-Pacific Comput. Syst. Architect. Conf., 2005, pp. $353-$ 366.

[19] S.-L. Lu, "Speeding up processing with approximation circuits," Computer, vol. 37, no. 3, pp. 67-73, Mar. 2004.

[20] Y. V. Ivanov and C. J. Bleakley, "Real-time h.264 video encoding in software with fast mode decision and dynamic complexity control," ACM Trans. Multimedia Comput. Commun. Applicat., vol. 6, pp. 5:1-5:21, Feb. 2010.

[21] M. Shafique, L. Bauer, and J. Henkel, "enBudget: A run-time adaptive predictive energy-budgeting scheme for energy-aware motion estimation in H.264/MPEG-4 AVC video encoder," in Proc. Design, Automat. Test Eur., Mar. 2010, pp. 1725-1730.

[22] V. Gupta, D. Mohapatra, S. P. Park, A. Raghunathan, and K. Roy, "IMPACT: Imprecise adders for low-power approximate computing," in Proc. IEEE/ACM Int. Symp. Low-Power Electron. Design, Aug. 2011, pp. 409-414.

[23] J. M. Rabaey, Digital Integrated Circuits: A Design Perspective. Upper Saddle River, NJ: Prentice-Hall, 1996.

[24] [24] E. Lyons, V. Ganti, R. Goldman, V. Melikyan, and H. Mahmoodi, "Full-custom design project for digital VLSI and IC design courses using synopsys generic 90nm CMOS library," in Proc. IEEE Int. Conf. Microelectron. Syst. Edu., Jul. 2009, pp. 4548 . 